\title{
Revisão de esfregaços cervicais negativos em pacientes com lesões intra-epiteliais de alto grau
}

Review of previous negative smears from patients with high grade intraepithelial neoplasia

\author{
Janaína Erika Pittoli ${ }^{1}$ \\ Evandro Sobroza de Mello ${ }^{1}$ \\ Sonia Maria Miranda Pereira ${ }^{1}$ \\ Marina Yoshiê Sakamoto Maeda ${ }^{1}$ \\ Maria Lucia Utagawa ${ }^{1}$ \\ José Donizete Celestino \\ Celso di Loreto ${ }^{1}$
}

\section{unitermos resumo}

Lesão intra-epitelial de

Entre as estratégias de controle de qualidade, a revisão de exames negativos anteriores em

alto grau

pacientes com exame atual positivo é considerada uma das mais eficientes para detecção de

falsos negativos. No presente estudo foram revistos os esfregaços prévios negativos de

pacientes com NIC 2 ou NIC 3 nos anos de 1998 e 1999. Durante este período o Instituto

Controle de qualidade

Adolfo Lutz realizou 113.619 exames colpocitológicos, sendo 233 casos diagnosticados como

Citologia cervicovaginal

NIC 2 ou NIC 3. Entre estes casos apenas 36 tinham exames prévios no Instituto Adolfo

Lutz no período de 36 meses antes do diagnóstico original. Obtivemos 22 casos de NIC

(12 NIC 1, sete NIC 2 e três NIC 3) e 14 casos com diagnóstico negativo (normal ou

inflamatório), os quais pertenciam a 12 pacientes. Após revisão destes esfregaços obtivemos os seguintes resultados: insatisfatório (dois casos), inflamatório (cinco casos), NIC 1

(três casos), NIC 2 (um caso). Concluindo, a revisão dos exames anteriores negativos é uma estratégia altamente eficiente para detectar os resultados falsos negativos. Sua prática pode ser útil para orientar diversas medidas de educação continuada.

\section{abstract key words}

Within the strategies of internal quality control, the review of previous negative Papanicolaou smears from patients with diagnosis of CIN 2 or CIN 3 is considered one of the most efficient for detection of false negative results. In the present study we reviewed the previous negative smears of cases with diagnosis of CIN 2 or CIN 3 during the years of 1998 and 1999. During this period the Adolfo Lutz Institute has performed 113.619 Papanicolaou tests; 233 cases were diagnosed

as CIN 2 or CIN 3. The cases diagnosed as unsatisfactory were excluded. Among the 36 cases from which there were previous smears in the period of 36 months before the original diagnosis, we found 22 cases of CIN (12 CIN 1, seven CIN 2 and three CIN 3) and 14 cases diagnosed as inflammatory or normal. When those 14 smears were reviewed, the following diagnosis were found: unsatisfactory (two cases), inflammatory (five cases), Ascus (three cases), CIN 1 (three cases), CIN 2 (one case). In conclusion, the review of previous negative Papanicolaou smears is a highly efficient strategy in order to detect false negative results. This practice can be useful as a guide for continued education measures.
High grade squamous lesion

Intraepithelial cervical

neoplasia

Quality control of cytopathology

Cervical smears 


\section{Introdução}

Todos os laboratórios de citopatologia têm a responsabilidade de manter um programa de qualidade, o qual deve basear-se no monitoramento contínuo de diversas estratégias de avaliação (6). Entre estas estratégias, a revisão crítica de exames colpocitológicos negativos anteriores de pacientes com diagnóstico atual de neoplasia intra-epitelial cervical grau 2 (NIC 2) ou neoplasia intraepitelial cervical grau 3 (NIC 3) é considerada por diversos autores um dos métodos mais eficientes para detecção de falsos negativos $(1,8)$. O objetivo deste estudo foi avaliar as dificuldades para aplicação e a eficiência desta estratégia na detecção de casos falsos negativos, além de identificar e analisar os fatores que podem causar tais erros.

\section{Material e método}

No período 1998-1999 foram analisados, no laboratório de citopatologia do Instituto Adolfo Lutz (IAL), 113.619 exames colpocitológicos, sendo que 233 casos foram diagnosticados como NIC 2 ou NIC 3.

Entre estes casos, apenas 36 tinham citologias anteriores no IAL no período de 36 meses antes do diagnóstico de NIC 2 ou NIC 3, e, destes, 22 casos tinham citologia anterior de NIC (12 NIC 1, sete NIC 2 e três NIC 3) e 14 tinham diagnóstico negativo (normal ou inflamatório). Para obtenção dessas informações foi realizado levantamento manual em nossos arquivos e levantamento de prontuários das pacientes junto às unidades de saúde. As lâminas correspondentes a estes casos foram submetidas a escrutínio por dois observadores independentes e posteriormente foram revistas em microscópio de múltipla observação, quando foi feito diagnóstico por dois patologistas (CL e ESM).

Revisão dos 14 exames colpocitológicos com citologia

Tabela anterior normal ou inflamatória

\begin{tabular}{lc}
\hline Resultado da revisão & № \\
Insatisfatório & 2 \\
Inflamatório & 5 \\
Ascus & 3 \\
NIC 1 & 3 \\
NIC 2 & 1 \\
\hline
\end{tabular}

\section{Resultados}

Dos 14 casos revistos, cinco mantiveram o diagnóstico original (inflamatório). Destes, dois casos apresentaram limitação por ausência de representação da junção escamocolunar (JEC) e dois casos por ressecamento parcial. Os nove casos restantes tiveram os diagnósticos modificados: dois foram reclassificados como inadequados devido a exsudato purulento; três como Ascus; três como NIC 1 e um como NIC 2. Todos os casos reclassificados como Ascus, NIC 1 ou NIC 2, na revisão, tinham número pequeno de células alteradas.

\section{Discussão}

Diversos autores têm destacado a importância da revisão de exames anteriores negativos em casos atuais positivos como estratégia para monitoramento da qualidade $(2,3,5,7)$. Nos EUA a legislação do CLIA-92 (6) recomenda a revisão dos esfregaços nos cinco anos prévios. Allen e Tabbara (8) ressaltam que a revisão de três anos consegue recuperar $94 \%$ dos casos anteriores negativos, sendo portanto mais adequada esta estratégia de revisão. Em nosso trabalho seguimos esta orientação e revimos apenas os casos correspondentes aos 36 meses anteriores. Concluímos com base nos resultados acima que esta estratégia pode ser eficiente para detectar um grupo de casos com elevada proporção de diagnósticos incorretos. Se considerarmos falsos negativos apenas os casos reclassificados como NIC teremos um total de quatro casos em 14 (28\%), valores semelhantes aos relatados por Tabbara e Sidawy (8) (38\%), Rohr (5) (21,7\%) e Sherman e Kelly (7) (22,7\%). Entre os nove esfregaços que tiveram os diagnósticos por nós modificados, dois foram reclassificados como inadequados devido a exsudato purulento. Este fato ressalta a importância da correta classificação dos esfregaços obscurecidos por hemorragia, exsudato purulento ou artefatos causados por fixação inadequada. Comparando esfregaços corretamente diagnosticados como NIC 2 ou NIC 3 com outros casos de NIC 2 ou NIC 3 que foram incorretamente diagnosticados como negativos, em reescrutínio rápido, O'Sullivan et al. (4) observaram que estes últimos tinham, em geral, menor quantidade de células displásicas, sendo que em muitas ocasiões tais células apresentavam núcleos mais pálidos. Em nossos achados, os casos reavaliados que apresentavam lesão precursora também tinham pequeno número de células alteradas. 
A revisão crítica destas amostras é um exercício eficiente de educação continuada e permite entender melhor as causas de diagnósticos incorretos, bem como planejar formas de melhorar o desempenho do laboratório. Embora represente uma estratégia eficiente, acreditamos que o presente levantamento, pelo número de horas de trabaIho que consumiu de nossa equipe, deveria ter conseguido resgatar um maior número de casos. Investimentos em informatização têm permitido mais recentemente a pesquisa imediata de eventuais exames citológicos anteriores e a correlação citoistológica, atividades fundamentais para monitoramento da qualidade. Entretanto nem sempre as requisições são preenchidas adequadamente. Muitas vezes a identificação destas pacientes é prejudicada pela mudança ou abreviação de sobrenomes. Outro problema importante é o envio de amostras para diferentes laboratórios, dificultando a revisão de exames anteriores. Embora este último aspecto seja de difícil resolução, acreditamos que, nos laboratórios públicos, a adoção de um registro centralizado destes diagnósticos de acordo com um código único por paciente e uma organização mais eficiente nos sistemas de referência e contra-referência, bem como investimento em informatização, seriam importantes avanços para a qualidade.

\section{Referências}

1. Allen, K.A.; Zaleski, S. \& Cohen, M.B. Review of negative Papanicolaou tests. Is the retrospective 5-year review necessary? Am. J. Clin. Pathol., 101: 19-21, 1994.

2. Jones, B.A. Rescreening in gynecolo gic cytology. Arch. Pathol. Lab. Med., 119: 1097-103, 1995.

3. Mody, D.R. \& Davey D.D. et al. Q uality assurance and risk reduction guidelines. Acta Cytologica, 44:496-507, 2000.

4. 0 'Sullivan, J.P. et al. Characteristics of high grade dyscaryotic cervical smears likely to be missed on rapid rescreening. Acta Cytologica, 44: 37-40, 2000.
5. Rohr, L.R. Q uality assurance in gynecologic cytology. Am. J. Clin Pathol., 94: 754-8,1990.

6. Rules and Regulations. Federal Register, 57: 7137, 1992.

7. Sherman, M.E. \& Kelly, D. H igh-grade squamous intraepithelial lesions and invasive carcinoma following the report of three negative Papanicolaou smears: screening failures or rapid progression? M od. Pathol. 5: 337-42, 1992.

8. Tabbara, S.O . \& Sidawy, M.K. Evaluation of the 5-year review of negative cervical smears in patients with high grade squamous intraepithelial lesions. Diagn. Cytopathol., 15: 7-11, 1996.
Endereço para correspondência

Celso di Loreto

Av. Dr. Arnaldo 351/70 andar - Cerqueira César CEP 01246-902 - São Paulo-SP

Fax: (21) 3085-3505 